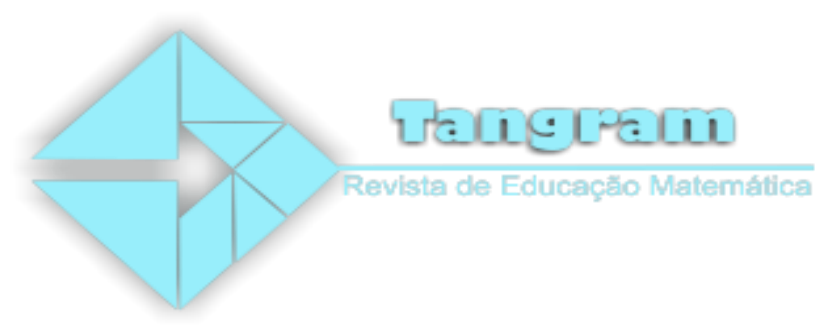

\title{
BNCC do ensino médio: um olhar sobre os conteúdos de área e volume nos livros didáticos de matemática
}

\section{High school's BNCC: a view regarding the contents of area and volume on math textbooks}

\author{
Brena Cristina Sturion ${ }^{1}$
}

Rúbia Barcelos Amaral-Schio ${ }^{2}$

\begin{abstract}
Resumo: O presente trabalho é fruto de uma pesquisa qualitativa realizada a partir da análise de três coleções de livros didáticos de Matemática de Ensino Médio, aprovados pelo PNLD 2018. O foco de estudo esteve nos conteúdos de área e volume, e para a análise foi considerado como referencial teórico o conhecimento especializado do professor de Matemática. Para discussão no evento, nosso objetivo é trazer os resultados que tangem à apreciação desses conceitos à luz da Base Nacional Comum Curricular (BNCC). Após uma análise documental pudemos perceber que, ainda que os livros tenham sido escritos antes da publicação da BNCC, a maior parte das habilidades sugeridas por esse documento é contemplada no desenvolvimento desses conteúdos.
\end{abstract}

Palavras-chave: Base Nacional Comum Curricular; Figuras planas; Corpos redondos; Poliedros; MTSK.

\begin{abstract}
This paper is a result of a qualitative research conducted from the analysis of three collections of high school math textbooks, applied by PNLD 2018. The focus of the study was presented in the contents of the area and volume, and for an analysis was considered as theoretical reference or specialized knowledge of the mathematics teacher. For event discussion, our goal is to bring the results that tangent these concepts in light of the BNCC (Common National Curriculum Base). After a documentary analysis, we realized that, although the books were written before the publication of the BNCC, most of the skills suggested by this document are contemplated in the development of these contents.
\end{abstract}

Keyword: Volume. Polyhedron. MTSK.

\footnotetext{
${ }^{1}$ Graduanda em Matemática, UNESP - Rio Claro/SP. E-mail: sturionbrena@gmail.com

${ }^{2}$ Livre-Docente em Educação Matemática. Departamento de Educação Matemática. Unesp - Rio Claro/SP.

E-mail: rubia.amaral@unesp.br
}

Tangram - Revista de Educação Matemática, Dourados - MS - v.2 n. 3, pp. 88-102 (2019) 
BNCC do ensino médio: um olhar sobre os conteúdos de área e volume nos livros didáticos de matemática

\section{Introdução}

O presente texto é fruto de uma pesquisa desenvolvida pela primeira autora, com orientação da segunda. $\mathrm{O}$ foco dessa pesquisa esteve nos conteúdos de área e volume presentes em três coleções de livros didáticos do Ensino Médio, aprovadas pelo PNLD 2018. Ao estudar esses conteúdos foi realizada uma análise das figuras planas, bem como dos poliedros e corpos redondos. Um dos temas de análise centrou-se no estudo da BNCC, buscando entender quais habilidades são esperadas do aluno quando do estudo de área e volume. A análise considerou como lente teórica o conhecimento especializado do professor de Matemática, a qual selecionamos para compartilhar e discutir no evento.

Nesse sentido, trazemos as perspectivas que embasam o conceito teórico sobre o conhecimento especializado do professor de Matemática, o qual alguns de seus subdomínios são usados para interpretar os dados construídos a partir da análise documental dos livros. As habilidades tratadas na BNCC sobre área e volume são apresentadas na sequência, juntamente a uma análise dos livros, considerando também alguns exemplos de atividades neles incluídas. Fechamos, por fim, com algumas reflexões que ficam em aberto para pesquisas futuras sobre a temática aqui abordada.

\section{Conhecimento especializado do professor de Matemática}

O Mathematics Teacher's Specialised Knowledge (MTSK) apareceu como uma solução para os problemas que o modelo de Ball, Thames, e Phelps (2008), Mathematical Knowledge Teaching (MKT), apresentava. Este mostrava dificuldades nas delimitações e nas características de cada uma de suas diversas categorias (FLORES-MEDRANO et al., 2014).

O MTSK considera, de maneira integral, o conhecimento do professor em todas as suas subdivisões, sem se referenciar com algo externo. Além disso, ele mantém a divisão estabelecida por Shulman (1986), que também foi referencial de Ball, Thames, e Phelps (2008), sobre os domínios do conhecimento, que são: Conhecimento do Conteúdo e o Conhecimento Pedagógico do Conteúdo. A partir deles Flores-Medrano et al. (2014) centram-se em questões relacionadas à Matemática, dividindo-os em três subdomínios cada.

Tangram - Revista de Educação Matemática, Dourados - MS - v.2 n. 3, pp. 88-102 (2019) 
BNCC do ensino médio: um olhar sobre os conteúdos de área e volume nos livros didáticos de matemática

O que tange o primeiro domínio, Conhecimento do conteúdo ${ }^{3}(M K)$ é o conhecimento "científico" que o professor possui sobre a Matemática que ensina. Já que existe uma diferença entre o que um profissional da Educação Matemática conhece e o que outras pessoas possam saber de Matemática. O MK é dividido em três subdomínios: o conhecimento dos temas matemáticos ${ }^{4}$ (KoT), o conhecimento da estrutura matemática ${ }^{5}$ (KSM), e o conhecimento da prática matemática ${ }^{6}(\mathrm{KPM})$.

Sobre o KoT, este engloba o conhecimento acerca dos conteúdos matemáticos e seus significados de forma fundamentada, integrando o conhecimento que está programado para que o aluno aprenda, considerando também um saber um pouco mais profundo que o passado em sala de aula. Ele é subdividido em cinco categorias. A primeira delas é a fenomenologia, que possui um caráter duplo. Por um lado, considera o conhecimento a respeito dos modelos atribuídos a um determinado tema como sendo fenômenos que podem gerar o saber matemático. Por outro lado, pondera o conhecimento que o uso e as aplicações de um tema proporcionam. A segunda categoria preza pelo conhecimento das propriedades e de seus fundamentos de um tópico em particular. A terceira são os registros e representações, esta tange o conhecimento de formas distintas de apresentar um conteúdo e o vocabulário, ou notação, adequado para cada representação. A quarta são as definições, que se relacionam com o saber que o professor tem sobre as propriedades que fazem um objeto ser definível. E, por último, a quinta categoria, os procedimentos, tange o conhecimento sobre os algoritmos, convencionais ou não, as condições para proceder, os fundamentos de tais algoritmos e as características do resultado do tema em questão.

Continuando com os subdomínios do MK, o conhecimento da estrutura matemática (KSM) trata do conhecimento que o profissional da Educação Matemática tem sobre as relações entre distintos tópicos, ou seja, as conexões entre os conteúdos matemáticos. Ele está subdividido em quatro categorias. A primeira é conexões de complexidade, que visa relacionar o que está sendo ensinado com os conteúdos que serão abordados posteriormente. A segunda é conexões de simplificação, que busca associar os conteúdos atuais com os que

\footnotetext{
${ }^{3}$ Mathematical Knowledge - MK.

${ }^{4}$ Knowledge of Topics - KoT.

${ }^{5}$ Knowledge of the Structure of Mathematicks - KSM.

${ }^{6}$ Knowledge of Practices in Mathematics - KPM.
}

Tangram - Revista de Educação Matemática, Dourados - MS - v.2 n. 3, pp. 88-102 (2019) 
BNCC do ensino médio: um olhar sobre os conteúdos de área e volume nos livros didáticos de matemática

já foram vistos anteriormente. A terceira, conexões de conteúdos transversais, engloba as características comuns que entrelaçam as duas categorias citadas acima. Por fim, a quarta, conexões auxiliares, faz alusão à quando o professor usa de um conteúdo para auxiliar na resolução de problemas, ou na explicação, de um conteúdo distinto deste.

O último subdomínio do MK é o conhecimento da prática matemática (KPM), que trata do professor não só ter o conhecimento dos resultados matemáticos, mas também dos processos para se chegar a eles. Este se divide em duas categorias. Primeiramente temos as práticas ligadas à Matemática em geral, que considera como se explora e se desenvolve o conhecimento da Matemática, independente do conceito abordado, o que ajuda a entender o funcionamento dos diferentes aspectos matemáticos. A segunda categoria, das práticas ligadas à uma temática matemática, abordam as mesmas ideias anteriores, porém voltadas para um tópico em específico.

Referindo-se ao segundo domínio do conhecimento, o conhecimento pedagógico do conteúdo ${ }^{7}(P C K)$, ele é algo intrínseco do professor, próprio do trabalho de ensinar.

A inclusão de aspectos correspondentes ao PCK no MTSK reconhece a importância de que o professor conheça o conteúdo matemático desde o ponto de vista de um conteúdo a ser ensinado [...], passando por um ponto de vista do conteúdo a ser aprendido [...], até uma visão geral dos padrões que se pretende alcançar (Flores-Medrano et al. 2014. p.79).

Este domínio está dividido em três subdomínios: o conhecimento das características de aprendizagem $^{8}$ (KFLM), o conhecimento do ensino de Matemática ${ }^{9}$ (KMT) e o conhecimento das normas de aprendizagem matemática ${ }^{10}$ (KMLS).

O KFLM trata do conhecimento das características de aprendizagem próprias do conteúdo matemático, ou seja, do saber relacionado com as características derivadas da interação do aluno com o conteúdo. Este subdomínio está dividido em quatro categorias.

A primeira refere-se às formas de aprendizagem, que é o saber que o professor tem sobre os possíveis modos de compreensão associados ao tema estudado, incluindo o conhecimento de teorias pessoais sobre o desenvolvimento cognitivo do aluno. A segunda

\footnotetext{
${ }^{7}$ Pedagogical Content Knowledge - PCK.

${ }^{8}$ Knowledge of Features of Learning Mathematics - KFLM.

${ }^{9}$ Knowledge Mathematics of Teaching - KMT.

${ }^{10}$ Knowledge of Mathematics Learning of Standards - KMLS.

Tangram - Revista de Educação Matemática, Dourados - MS - v.2 n. 3, pp. 88-102 (2019)
} 
BNCC do ensino médio: um olhar sobre os conteúdos de área e volume nos livros didáticos de matemática

tange a facilidade e dificuldade no aprendizado, englobando o conhecimento sobre os erros, obstáculos e dificuldades relacionados com os conteúdos matemáticos, mas pensando também no enriquecimento da aprendizagem, quando o professor trabalhar esses desafios. A terceira categoria aborda as formas de interação entre o aluno e o conteúdo de Matemática, referindo-se ao conhecimento do professor sobre os métodos e estratégias dos estudantes, sejam incomuns ou não, para compreender os temas dados em aula, além de se atentar ao linguajar usado na abordagem do conteúdo, o que pode auxiliar, ou não, o aprendizado por parte dos alunos. Por fim, a quarta categoria trata da concepção dos estudantes sobre a Matemática, que é o conhecimento do docente sobre o interesse que os estudantes têm em Matemática, a percepção das dificuldades e/ou facilidades dos alunos com determinada área da disciplina.

No que tange o segundo subdomínio do PCK, o conhecimento do ensino de matemática (KMT), ele considera o conhecimento de recursos, materiais, modos de abordagem e o poder que uma boa instrução pode ter, assim como conhecer os exemplos e contextos adequados para cada tópico. Ele está dividido em três categorias.

A primeira, teorias pessoais e institucionalizadas de ensino, tem em vista o poder que certas atividades, estratégias e processos didáticos associados à um conteúdo tem. Além de saber sobre exemplos, explicações e outros métodos que ajudam na abordagem do conteúdo. A segunda relaciona-se aos recursos materiais e visuais, e engloba o conhecimento do professor acerca dos recursos como elementos de ensino, os benefícios e dificuldades de seu uso como apoio na abordagem de determinado conteúdo, não tendo em vista uma ferramenta pedagógica, mas um complemento que auxilia na compreensão do aluno. A terceira abrange as atividades, tarefas, exemplos e ajudas. Difere da anterior que diz respeito ao conhecimento do professor para com o recurso. Esta categoria se preocupa com o momento certo, qual o tipo de ajuda, ou recurso usar, quais os melhores exemplos e qual o interesse da sala em conhecer mais sobre determinado tema.

O último subdomínio do PCK é o conhecimento das normas de aprendizagem Matemática (KMLS), que visa o conhecimento do docente sobre os conteúdos preestabelecidos que devem ser ensinados para determinado nível escolar. "Entendemos como normas de aprendizagem aquilo que indica o nível de capacidade atribuído aos

Tangram - Revista de Educação Matemática, Dourados - MS - v.2 n. 3, pp. 88-102 (2019) 
BNCC do ensino médio: um olhar sobre os conteúdos de área e volume nos livros didáticos de matemática

estudantes em um determinado momento escolar - para entender, construir e saber Matemática" (FLORES-MEDRANO et al., 2014, p.83, tradução nossa). Este está dividido em três categorias.

A primeira são os conhecimentos matemáticos que devem ser ensinados, que trata do conhecimento do professor dos temas que devem ser abordados no nível/ano que ele está trabalhando, assim como a maneira que o professor escolhe para alcançar esse fim. A segunda é o conhecimento do nível de desenvolvimento conceitual e processual esperado, que diz a respeito ao conhecimento do docente sobre os níveis de abstrações e complexidade que os alunos devem entender em determinado momento escolar. Por fim, a terceira categoria focaliza o sequenciamento de diversos tópicos, versando sobre os conhecimentos prévios que o aluno possui e aqueles que deve aprender para o entendimento dos conteúdos dos níveis posteriores. Esse subdomínio, basicamente, engloba o saber do professor acerca do que o aluno deve/pode aprender no nível em que está, além das capacidades conceituais, de processos e raciocínio que se promovem em cada momento escolar.

Os autores consideram também as concepções que os docentes possuem acerca da Matemática, seu ensino e aprendizagem, pois estas permeiam o conhecimento apresentado em cada subdomínio. Essas "crenças" são duas: na Matemática e no ensino e aprendizagem de Matemática, conseguindo com isso um cenário mais preciso para interpretar a prática docente e os aspectos que influenciam e embasam os conhecimentos que sustentam tal prática.

\section{Aspectos Metodológicos}

A pesquisa que apresentamos foi desenvolvida segundo uma abordagem interpretativa de pesquisa qualitativa. Como afirmam Bogdan e Biklen (1994, p.209), os estudos dessa natureza “devem revelar maior preocupação pelo processo e significado e não pelas suas causas e efeitos”. E, nesse sentido, o foco esteve nos conteúdos de área e volume, à luz da BNCC do Ensino Médio, sob uma interpretação cuja lente teórica era o conhecimento especializado do professor de Matemática, e não em seus resultados ou produtos, o que contempla uma das características centrais da pesquisa qualitativa, segundo Denzin e Lincoln (2000) e Vidich e Lyman (2000).

Tangram - Revista de Educação Matemática, Dourados - MS - v.2 n. 3, pp. 88-102 (2019) 
BNCC do ensino médio: um olhar sobre os conteúdos de área e volume nos livros didáticos de matemática

Para tal análise, foram considerados os conteúdos de área e volume, presentes na exploração de figuras planas, poliedros e corpos redondos, em três coleções de livros didáticos destinados ao Ensino Médio, aprovados pelo PNLD 2018: Conexões com a Matemática (Leonardo, 2016), Matemática: Ciência e Aplicações (Iezzi et al., 2017), Contato Matemática (Souza; Garcia, 2016). Vale ressaltar ainda, segundo Alves-Mazzotti (2001, p.131), que "a principal característica das pesquisas qualitativas é o fato de que estas seguem uma tradição 'compreensiva' ou interpretativa”. Além disso, Bogdan e Biklen (1994) orientam a estudar objetivamente as questões subjetivas inerentes à pesquisa. E, para tanto, alertam que é importante a seleção de métodos que "limitem" substancialmente o viés do envolvimento do pesquisador, apesar deste nunca ser totalmente "eliminado". Uma sugestão, segundo esses autores, é trabalhar em grupo, compartilhando os dados para análise e crítica dos colegas, como uma forma de proteção a esse enviesamento. Nesse sentido cabe observar que o processo de análise foi compartilhado com o GPIMEM - Grupo de Pesquisa em Informática, outras Mídias e Educação Matemática, da Unesp, cujos resultados estão em harmonia com a opinião dos autores, pois fomentou a "análise por pares" (LINCOLN; GUBA, 1985), considerando a colaboração para o estudo profundo dos aspectos teóricos, bem como dos objetivos da pesquisa.

\section{Descrição e Análise dos Dados}

Como descrevemos anteriormente, dentre os subdomínios do conhecimento pedagógico do conteúdo (PCK), há um voltado para o saber que o docente tem sobre as normas que regem o ensino da Matemática, o conhecimento das normas de aprendizagem Matemática $(K M L S)$. Este destaca o conhecimento que o professor dispõe sobre os conteúdos preestabelecidos por certas normas para um determinado nível escolar. "Entendemos como normas de aprendizagem aquilo que indica o nível de capacidade que os estudantes possuem em um determinado momento escolar para entender, construir e saber Matemática" (Flores-Medrano et al., 2014, p.83, tradução nossa).

Essas noções de níveis de capacidade são construídas pelo docente através do contato deste com algumas fontes sobre o currículo a ser ensinado em uma escola específica ou de maneira nacional, como é o caso da Base Nacional Comum Curricular (BNCC).

Tangram - Revista de Educação Matemática, Dourados - MS - v.2 n. 3, pp. 88-102 (2019) 
BNCC do ensino médio: um olhar sobre os conteúdos de área e volume nos livros didáticos de matemática

A BNCC é um documento que visa nortear o que é ensinado nas escolas brasileiras, compreendendo todas as etapas da Educação Básica, desde a Educação Infantil até o final do Ensino Médio. Trata-se de uma referência dos objetivos de aprendizagem de cada uma das fases de formação. A Base Nacional é uma ferramenta para orientar a elaboração do currículo específico de cada escola, sem desconsiderar as particularidades metodológicas, sociais e regionais de cada uma.

A BNCC para a Educação Infantil e o Ensino Fundamental foi aprovada e homologada em dezembro de 2017. Porém o foco desde texto é a BNCC voltada ao Ensino Médio. Esta, por sua vez, foi apresentada e encaminhada ao Conselho Nacional de Educação (CNE) em abril de 2018 e aprovada em dezembro do mesmo ano.

A Base do Ensino Médio se organiza da seguinte maneira: há quatro áreas do conhecimento, e na apresentação de cada uma delas existe um texto explicitando o seu papel na formação integral dos estudantes. Além disso, cada área estabelece competências específicas, cujo desenvolvimento deve ser promovido ao longo dessa etapa. Tais competências estão articuladas ao que foi estabelecido para o Ensino Fundamental, com as adequações necessárias ao atendimento das especificidades de formação dos estudantes do Ensino Médio. Por fim, para garantir o desenvolvimento das competências específicas das áreas, a cada uma delas é relacionado um conjunto de habilidades, que representam as aprendizagens essenciais a serem garantidas no âmbito da BNCC a todos os estudantes do Ensino Médio.

Considerando a parte específica de Matemática do documento, este apresenta cinco competências, sendo que os tópicos de áreas de figuras planas, poliedros e corpos redondos (tema do nosso estudo) são citados em três delas.

A primeira competência é a que propõe que os alunos devem adquirir conhecimentos matemáticos para interagir com o mundo contemporâneo, com base na análise de problemas cotidianos, recorrendo a conceitos e procedimentos próprios da Matemática, tomando, assim, decisões éticas e socialmente responsáveis. Para tal, a Base expõe a habilidade de "propor ações comunitárias, como as voltadas aos locais de moradia dos estudantes dentre outras, envolvendo cálculos das medidas de área, de volume, de capacidade ou de massa, adequados às demandas da região" (Brasil 2017, p.526)

Tangram - Revista de Educação Matemática, Dourados - MS - v.2 n. 3, pp. 88-102 (2019) 
BNCC do ensino médio: um olhar sobre os conteúdos de área e volume nos livros didáticos de matemática

A próxima competência recomenda que os alunos, por meio de atividades que exigem diferentes níveis de interpretação, utilizem dos meios matemáticos para interpretar e construir modelos que possam ser aplicados de modo a fundamentar a argumentação sobre os resultados dos problemas. A parte desta que cabe às áreas de figuras planas e poliedros foi identificada em duas habilidades. A primeira delas é:

Empregar diferentes métodos para a obtenção da medida da área de uma superfície (reconfigurações, aproximação por cortes etc.) e deduzir expressões de cálculo para aplicá-las em situações reais, como o remanejamento e a distribuição de plantações, com ou sem apoio de tecnologias digitais (Brasil, 2017, p.528).

Para trabalhar esta recomendação, é provável que o docente sentirá a necessidade de recorrer a outros materiais além do livro didático, pois não é sempre que as diversas opções de tipo de cálculo estão disponíveis em um único livro. Considerando as três coleções que analisamos, a figura geométrica que é mais comum os livros abordarem formas distintas do cálculo de área, tanto a fórmula como o procedimento, é o triangulo. Além da mais comum, onde sua área é dada pela metade do produto da base pela altura, nos é apresentado como obtê-la em função de dois lados da figura e do ângulo entre eles, ou até com o seu semiperímetro, a partir da Fórmula de Herão, ou Heron.

Para as outras figuras, o que se percebe com maior frequência é a apresentação de mais de uma maneira de dedução da fórmula e não do cálculo, ou seja, nos é exposto um modo distinto de procedimento que resulta na mesma fórmula.

Um exemplo dessa outra maneira de dedução é encontrada em Leonardo (2016), onde o método principal que resulta na área do trapézio é por meio da soma das áreas de triângulos. Logo ao lado, ele expõe uma maneira de dedução alternativa, partindo de dois trapézios congruentes e justapondo-os para formar um paralelogramo (Figura 1).

Tangram - Revista de Educação Matemática, Dourados - MS - v.2 n. 3, pp. 88-102 (2019) 
BNCC do ensino médio: um olhar sobre os conteúdos de área e volume nos livros didáticos de matemática

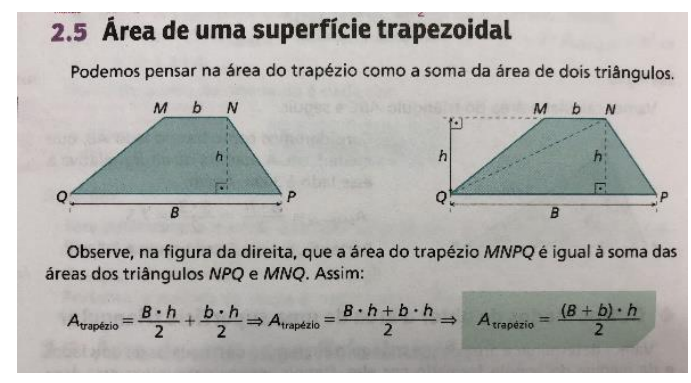

\section{\& Explore}

Experimente obter a área de um trapézio por meio de um procedimento diferente desse que foi exposto ao lado. Por exemplo, em um papel, desenhe e recorte dois trapézios MNPQ iguais. Justaponha-os de modo que formem um paralelogramo. Em seguida, por meio da fórmula da área do paralelogramo obtido, consiga a fórmula da área do trapézio $M N P Q$.

Figura 1 - Área do trapézio

Fonte: Leonardo (2016, p.70)

A segunda habilidade é assim proposta:

Resolver e elaborar problemas que envolvem o cálculo de áreas totais e de volumes de prismas, pirâmides e corpos redondos (cilindro e cone) em situações reais, como o cálculo do gasto de material para forrações ou pinturas de objetos cujos formatos sejam composições dos sólidos estudado. (Brasil, 2017, p.529).

Uma das maneiras que o professor encontra para trabalhar essa habilidade com os alunos é por meio de exercícios contextualizados, como os encontrados nos livros didáticos de Matemática. Olhando particularmente para as coleções que estão em análise neste texto, podemos exemplificar como tais exercícios se apresentam.

O livro de Souza e Garcia (2016) nos mostra um problema onde se deve calcular a quantidade de água que uma torneira gotejando desperdiça (Figura 2).

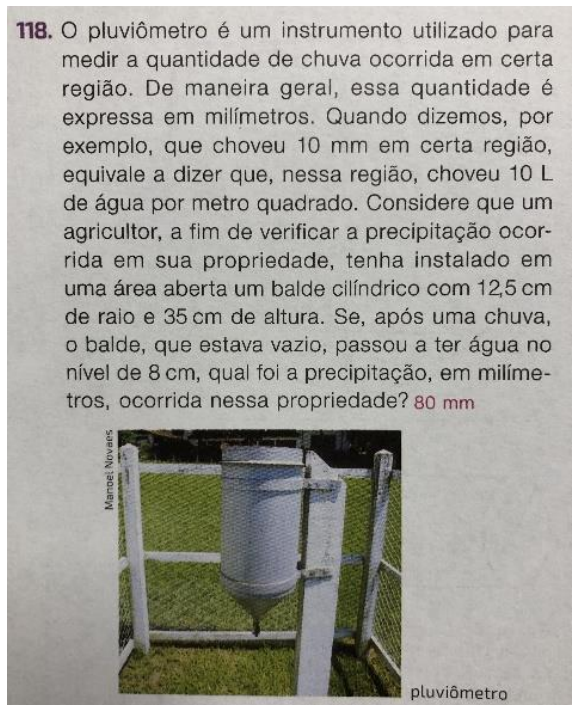

Figura 2 - Exercício de desperdício de água

Fonte: Souza e Garcia (2016, p.241) 
BNCC do ensino médio: um olhar sobre os conteúdos de área e volume nos livros didáticos de matemática

Já a obra de Iezzi et al. (2017) apresenta uma sala onde se deve colocar ladrilhos sem haver perda de material e sem saber as dimensões de tal ladrilho (Figura 3).

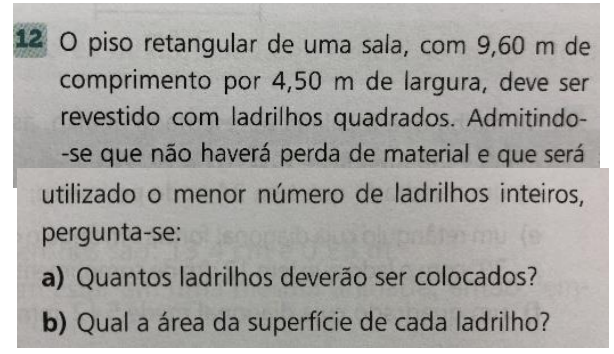

Figura 3 - Exercício do ladrilho

Fonte: Iezzi et al. (2017, p.232)

E como último exemplo temos no material de Leonardo (2016) que nos traz uma barra de prata que foi fundida em forma de prisma e precisa-se descobrir a massa (Figura 4).

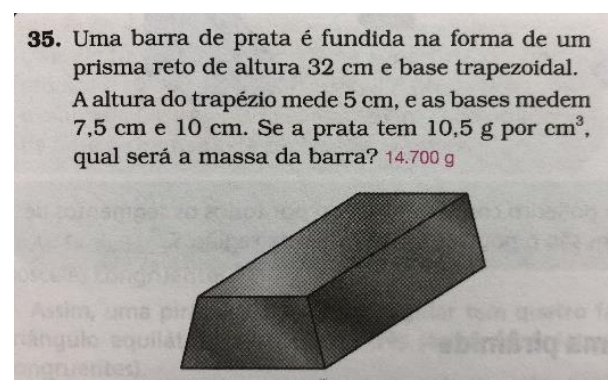

Figura 4 - Exercício barra de prata

Fonte: Leonardo (2016, p.119)

A partir de exercícios como os mostrados anteriormente, além do estudante ter de colocar em prática o que foi ensinado em sala de aula, o que por si só contribui para o melhor entendimento do tema, ele passa a perceber que a Matemática não é só uma disciplina fixada dentro das escolas, mas que ela pode ser encontrada facilmente no seu cotidiano. A ampliação do conhecimento sobre o conteúdo surge quando o aluno consegue notar que os cálculos matemáticos são uteis para situações diversas, como por exemplo, quantificar um desperdício de água e, a partir da interpretação do resultado, reparar que esta quantidade poderia estar sendo utilizada para algo mais produtivo. Além disso, muitas dessas situações já foram, ou serão, presenciadas pelo estudante, o que pode consolidar ainda mais seu conhecimento. Se foi algo já vivenciado por ele, pode acontecer dele associar o que é ensinado com as suas experiências pessoais. E se ele se deparar posteriormente com um cenário parecido com o mostrado em aula, este poderá o ajudar a entender melhor a situação.

Tangram - Revista de Educação Matemática, Dourados - MS - v.2 n. 3, pp. 88-102 (2019) 
BNCC do ensino médio: um olhar sobre os conteúdos de área e volume nos livros didáticos de matemática

Atividades contextualizadas mostram aos discentes que a Matemática não é tão abstrata como aparenta ser. Isto facilita o rompimento da barreira que muitos alunos têm com a Matemática, pois quando conseguimos notar que algo está inserido em nossa realidade a compreensão sobre isso se torna muito mais fácil e até prazerosa.

Para finalizar, como última competência que cita os tópicos de áreas de figuras planas, poliedros e corpos redondos, o documento nos mostra a Matemática como uma atividade sujeita a acertos e erros, como um processo de busca, questionamentos e contraexemplos. Assim, seria importante que o aluno tenha experiências relacionadas à prática de estabelecer hipóteses a respeito dos diversos conceitos e propriedades matemáticas, investigando argumentos para provar que ela está certa ou contraexemplos onde ela não é válida. Logo temos três habilidades que nos são interessantes:

[1] Investigar processos de obtenção da medida do volume de prismas, pirâmides, cilindros e cones, incluindo o princípio de Cavalieri, para a obtenção das fórmulas de cálculo da medida do volume dessas figuras.[...] [2] Resolver problemas sobre ladrilhamentos do plano, com ou sem apoio de aplicativos de geometria dinâmica, para conjecturar a respeito dos tipos ou composição de polígonos que podem ser utilizados, generalizando padrões observados. [...]

[3] Investigar propriedades de figuras geométricas, questionando suas conjecturas por meio da busca de contraexemplos, para refutá-las ou reconhecer a necessidade de sua demonstração para validação, como os teoremas relativos aos quadriláteros e triângulos (Brasil, 2017, p.533).

Tendo em vista o subdomínio KMLS, é importante para a aprendizagem do aluno que ele saiba como surgiram as fórmulas que usa, que elas têm um porquê e que podem ser obtidas através de provas visuais e até demonstrações, dentro dos limites da Educação Básica. Ou seja, é interessante que o professor conheça os níveis de abstrações e complexidade que podem ser trabalhados com o Ensino Médio, assim não extrapolando as explicações com conceitos muito formais nem deixando algo sem uma interpretação satisfatória, atingindo desse modo um melhor entendimento e desenvolvimento das habilidades citadas no documento.

As obras aqui vistas apresentam tais explicações em muitos pontos de suas abordagens. Por exemplo ao utilizar o Princípio de Cavalieri, depois de explicá-lo, para se obter as fórmulas para o cálculo do volume de prismas e cilindros. Além disso, fazem uma 
BNCC do ensino médio: um olhar sobre os conteúdos de área e volume nos livros didáticos de matemática

prova visual do porque a fórmula do volume de uma pirâmide é um terço do volume de um prisma, também mostrando esses resultados, de maneira análoga, para cones.

É válido ressaltar que os livros analisados neste texto são aprovados pelo PNLD de 2018 e a Base foi homologada em 2018, logo as coleções não teriam a obrigação de apresentar todas as competências e habilidades que o documento cita. Porém, após a análise da BNCC conjuntamente com as três coleções, acabamos por encontrar muitos dos aspectos que por ela explicitados nestes materiais, mostrando que a nova base curricular não se distancia do conteúdo que já é ensinado nas escolas nacionais, ao menos quando tratado dos tópicos de figuras planas, poliedros e corpos redondos. Além do mais, como a Base não explicita em qual ano se deve trabalhar as competências, os livros didáticos são um possível referencial para que o professor se guie e tenha o conhecimento dos temas que devem ser abordados no nível em que está trabalhando.

Por fim, com o docente sabendo do que a Base trata e propõe, é possível que ele tenha a consciência dos conhecimentos que seus alunos deveriam ter adquirido no ano anterior e o que eles devem aprender na etapa atual para que consigam um bom entendimento dos conteúdos nos níveis posteriores, que é destacado pelo KMLS.

Assim, temos que o livro didático é um auxiliar para que o professor consiga que seus alunos entrem em contato com os temas, em particular as áreas de figuras planas, poliedros e corpos redondos, e até adquiram as habilidades que a Base propõe como indispensáveis ao estudante do Ensino Médio.

\section{Considerações Finais}

No presente texto trazemos um recorte da pesquisa que desenvolvemos acerca dos conteúdos de área a volume em livros didáticos de Matemática destinados ao Ensino Médio. Um dos temas de análise centrou no estudo da BNCC, o qual selecionamos para compartilhar e discutir no evento, pois é um tema atual e relevante, além de pertinente ao público alvo do ENEM, de modo que esperamos que as discussões possam enriquecer a nós e aos demais participantes.

Abordar questões relacionadas à BNCC é um campo aberto à pesquisa. Sabemos, em especial, que os livros didáticos têm passado por uma reformulação para contemplar as Tangram - Revista de Educação Matemática, Dourados - MS - v.2 n. 3, pp. 88-102 (2019) 
BNCC do ensino médio: um olhar sobre os conteúdos de área e volume nos livros didáticos de matemática

orientações contidas nesse documento oficial e esse é um campo frutífero de pesquisa que merece atenção de professores e pesquisadores em Educação Matemática. Esperamos contribuir com essa temática e incitar outras pesquisas sobre o tema.

\section{Agradecimentos}

Agradecemos à Secretaria de Educação Superior - SESu/MEC pelo financiamento dessa pesquisa.

\section{Referências}

Alves-Mazzotti, A.J. (2001). O método nas Ciências Sociais. In: Alves-Mazzotti, A.J.; Gewandsznajder, F. O método nas Ciências Naturais e Sociais: Pesquisa Quantitativa e Qualitativa. São Paulo: Editora Pioneira. p.107-188.

Ball, D.L.; Thames, M.H.; Phelps, G. (2008). Content knowledge for teaching: What makes it special?. Journal of teacher education. 59 (5). p.389-407.

Bogdan, R. C.; Biklen, S. K. (1994). Investigação qualitativa em educação. Porto: Porto Editora.

Brasil, Ministério da Educação. (2017). Base Nacional Comum Curricular. http://basenacionalcomum.mec.gov.br/wpcontent/uploads/2018/06/BNCC_EnsinoMedio_embaixa_site_110518.pdf.

Denzin, N.K.; Lincoln, Y.S. (2000). The discipline and practice of qualitative research, In: Denzin, N.; Lincoln, Y.S. Handbook of qualitative research. Second edition. Londres: Sage publications.

Flores-Medrano, E.; Escudero-Ávila, D.; Montes, M.A.; Aguilar, A.; Carrillo, J. (2014). Nuestra modelación del conocimiento especializado del profesor de matemáticas, el MTSK. Un marco teórico para el conocimiento especializado del profesor de matemáticas, p.70-92.

Iezzi, G.; Dolce, O.; Degenszajn, D.; Périgo, R.; Almeida, N. (2017). Matemática: Ciência e Aplicações. $9^{\circ}$ ed. Vol. 1. São Paulo: Saraiva.

Leonardo, F. (2016). Conexões com a Matemática. $2^{\circ}$ ed. Vol. 2. São Paulo: Moderna.

Lincoln, I.S.; Guba, E.G. (1985). Naturalistic Inquiry. London: Sage publications.

Shulman, L.S. (1986). Those who understand: Knowledge growth in teaching. Educational researcher. 15 (2), p.4-14.

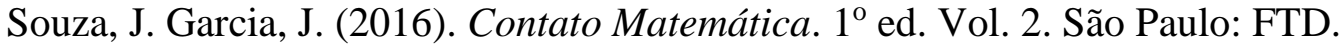
Tangram - Revista de Educação Matemática, Dourados - MS - v.2 n. 3, pp. 88-102 (2019) 
BNCC do ensino médio: um olhar sobre os conteúdos de área e volume nos livros didáticos de matemática

Vidich, A.J.; Lyman, S.M. (2000). Qualitative Methods: Their History in Sociology and Anthropology. In: Denzin, N.K.; Lincoln, I.S. (Org.). Handbook of Qualitative Research. 2.ed. London: Sage publications.

Enviado:26/09/2019

Aceito:14/10/2019

Tangram - Revista de Educação Matemática, Dourados - MS - v.2 n. 3, pp. 88-102 (2019) 